\title{
Comparison between the clinical efficacy of platelet concentrates, derived from buffy coat and apheresis in tumor patients
}

\author{
BAOLAN HAO, YAN WANG, JIAN ZHOU, SHUJUN SHAO and XIAOFENG DONG \\ Department of Blood Transfusion, The Affiliated Cancer Hospital of Zhengzhou University, \\ Zhengzhou, Henan 450008, P.R. China
}

Received March 2, 2017; Accepted May 12, 2017

DOI: $10.3892 / 01.2017 .6270$

\begin{abstract}
The aim of the present study was to evaluate the clinical efficacy between manual buffy coat-derived platelet concentrates (PCs) and automated apheresis platelet concentrates (APCs) in terms of their therapeutic effects. The corrected count increment (CCI) was calculated according to detected differences in platelet concentration in patients who underwent transfusion of APCs, prepared by an automated system (group I, 72 cases) or PCs derived from buffy coat by manual method (group II, 83 cases). The clinical efficacy was assessed in terms of the CCI and clinical symptoms. The platelet contents of all the PCs were detected before transfusion. The mean $1 \mathrm{~h} \mathrm{CCI}$ was $13.56 \pm 4.45$ and $24 \mathrm{~h} \mathrm{CCI}$ was $8.67 \pm 4.21$ in group I, while the mean $1 \mathrm{~h} \mathrm{CCI}$ was $15.83 \pm 4.65$ and $24 \mathrm{~h} \mathrm{CCI}$ was $9.57 \pm 3.36$ in group II. The effective rates judged by CCI for groups I and II were 53 and 64\%, respectively, and those judged by clinical symptoms were 67 and $60 \%$, respectively. In conclusion, the clinical effectiveness of manual PCs was similar to that for APCs; thus, it could be utilized for clinical use.
\end{abstract}

\section{Introduction}

In the Affiliated Cancer Hospital of Zhengzhou University, some patients would encounter thrombocytopenia due to their primary blood disease (such as leukemia and aplastic anemia) or marrow suppression caused by radiotherapy or chemotherapy. Low platelet (PLT) counts frequently lead to bleeding complications and even death, therefore platelet transfusion is a critical and often necessary aspect of managing cancer (1). Platelet transfusion is indicated either prophylactically or ther-

Correspondence to: Dr Yan Wang, Department of Blood Transfusion, The Affiliated Cancer Hospital of Zhengzhou University, 127 Dongming Road, Zhengzhou, Henan 450008, P.R. China

E-mail:ya73vm@163.com; 2528863571@qq.com

Key words: platelet, platelet concentrates, apheresis platelet concentrates, platelet transfusion efficiency apeutically to reduce the risk of bleeding, promote hemostasis or to control active bleeding (2). Platelet concentrates (PCs) are mainly produced by two different preparation methods: Manual PCs (collected from donated whole blood with the platelet-rich plasma-method or the buffy coat-method) and plateletpheresis (apheresis-derived platelet concentrates, APCs) (3). In our Henan Provincial Blood Center, manualPCs is produced by buffy coat-method. There is an ongoing debate whether manual PCs or plateletpheresis is superior. Manual PCs is isolated from donated whole blood that need multiple donations to have sufficient platelets to treat an adult patient, thereby increasing the risk of infectious agent transmission and alloimmunization rates compared with apheresis units that is collected from a single donor (4). So the APCs is always preferred over another in clinical settings but is always in short supply due to the increasing demand for medical treatment, resulting in manual PCs being frequently used.

In the present study, the clinical efficacy was observed in tumor patients to investigate the transfusion efficiency of the two types of platelet products.

\section{Patients and methods}

Patients. Patients with the following factors that may influence platelet count, such as fever, hepatosplenomegaly, bone marrow transplantation were excluded. All the subjects were divided into groups according to the two types of infused PCs. All the patients were Rh (D) positive and were transfused with ABO-matched PCs at a tolerant speed.

Group I (subjects transfused APCs) comprised 72 cases, and included 37 males and 35 females, with a mean age of 38.7 years (range, 7-76 years). Clinical diagnosis of the subjects was as follows: 50 cases of acute leukemia, 5 cases of endometrial carcinoma, 4 cases of gastric carcinoma, 4 cases of lymphoma, 2 cases of gallbladder carcinoma, 2 cases of osteosarcoma, 2 cases of ovarian cancer, 1 case was myelodysplastic syndrome (MDS) and 2 cases were myeloma. The platelet counts before transfusion were $3-41 \times 10^{9} / 1$ and the average count was $15.90 \times 10^{9} / 1$.

Group II (subjects transfused manually collected PCs) comprised 83 cases, including 48 males and 35 females, with a mean age of 41.8 years (range, 6-83 years). Clinical diagnosis of the subjects was as follows: 48 cases of acute leukemia, 6 cases of gastric carcinoma, 4 cases of breast cancer, 3 cases 
Table I. The platelet content of the two types of PCs.

\begin{tabular}{lcccc}
\hline Type & $\mathrm{n}(\mathrm{bag})$ & Net volume $(\mathrm{ml})$ & Net weight $(\mathrm{g})$ & Platelet count $\left(\mathrm{x} 10^{11}\right)$ \\
\hline APCs & 72 & $238 \pm 20.54$ & $250 \pm 31$ & $2.34 \pm 0.37$ \\
Manual PCs & 430 & $60.68 \pm 4.89$ & $63.05 \pm 4.79$ & $0.61 \pm 0.23$ \\
\hline
\end{tabular}

PCs, platelet concentrates; APCs, apheresis platelet concentrates.

Table II. The platelet counts (x10 $/ 1)$ of the two groups patients before and after transfusion.

\begin{tabular}{lcccr}
\hline Group & $\mathrm{n}$ & Before & After 1 h & After 24 h \\
\hline I & 72 & $15.90 \pm 7.67$ & $36.43 \pm 11.82$ & $31.28 \pm 10.40$ \\
II & 83 & $16.57 \pm 8.34$ & $42.63 \pm 13.26$ & $40.73 \pm 12.34$ \\
P-value & & 0.34 & 0.22 & 0.08 \\
\hline
\end{tabular}

Table III. CCI value and effective rate of transfusion in the two groups.

\begin{tabular}{lcccccc}
\hline Group & $\mathrm{n}$ & $1 \mathrm{~h} \mathrm{CCI}$ & $24 \mathrm{~h} \mathrm{CCI}$ & Valid cases & Invalid cases & Effective rate \\
\hline I & 72 & $13.56 \pm 4.45$ & $8.67 \pm 4.21$ & 38 & 34 & $53 \%$ \\
II & 83 & $15.83 \pm 4.65$ & $9.57 \pm 3.36$ & 53 & 30 & $64 \%$ \\
\hline
\end{tabular}

CCI, corrected count increment.

of ovarian cancer, endometrial carcinoma, lung cancer and MDS, respectively, 2 cases of esophageal cancer, 2 cases of cardiac cancer, 2 cases of rectal cancer, 2 cases of lymphoma, 1 case of liver cancer, 1 case of pancreatic carcinoma, 1 case of cervical cancer, 1 case of sarcoma of chest wall, and 1 case of vertebral tumor. The platelet counts before transfusion were $2-40 \times 10^{9} / 1$ and the average count was $16.57 \times 10^{9} / 1$.

Reagents. A total of 72 bags of APCs (a therapeutic dose/bag) and 430 bags of manual PCs ( 2 units/bag) were used in the present study. In China, the standard therapeutic adult dose is $>2.5 \times 10^{11}$ platelets and a unit dose is $>2.0 \times 10^{10}$ platelets. The APCs were prepared by the MCS + blood cell separator (produced by Haemonetics Corporation, Braintree, MA, USA). A balanced, bag heat-sealing machine, K-4500 hematology analyzer (East Asia Joint-Stock Co., Japan) was used in the experiment.

Platelet count of PCs. Weighed platelet products through sufficiently blending were transferred to a small tube in the bag. Then specimen was taken and mixed with normal saline at a ratio of 1:4 followed by detection of the platelet concentration. The platelet content was calculated using the equation: Platelet content $=($ gross weight - dry weight of the bag $) /$ relative density x platelet concentration $\left(\times 10^{9} / 1\right)$.

Efficacy judgment. The platelet transfusion efficiency was evaluated by corrected count increment (CCI), which is the most widely used marker for measuring the efficacy of platelet transfusions. CCI values were calculated using the formula: CCI $=$ increased PLA counting $\left(\times 10^{9} / 1\right) \times$ body surface area $\left(\mathrm{m}^{2}\right)$ /input PLA number $\left(\mathrm{x} 10^{11}\right)$. The standard of CCI $1 \mathrm{~h}$ $<7.5,24 \mathrm{~h}<4.5$ was accepted for invalid transfusion (1).

Observation of clinical symptoms. With the cooperation of clinicians and nurses, clinical symptoms of the patients before and after the transfusion were observed and noted to compare the efficacy of the two types of PCs.

Statistical analysis. Measurement data were presented as mean \pm standard deviation for statistical analysis. Data accorded with normal distribution were compared using the t-test, or rank-sum test (Mann-Whitney U test). Numeration data were statistically analyzed using the $\chi^{2}$ test. SPSS 21.0 statistical software (IBM SPSS, Armonk, NY, USA) was used for the statistical analysis. $\mathrm{P}<0.05$ was considered to indicate a statistically significant difference.

\section{Results}

The platelet content detection of all the PCs is provided in Table I. As can be seen from Table I, the mean platelet content of a bag of plateletpheresis was $2.34 \pm 0.37 \times 10^{11}$, and the mean platelet content of manual PCs from one donor was $0.61 \pm 0.23 \times 10^{11}$. On average, approximately four handmade platelets are equivalent to a bag of apheresis platelets. The 
platelet counts of the two groups before and after transfusion are provided in Table II. The mean value of the platelet counts in group II was a little higher than that in group I, but there was no statistical significance $(\mathrm{P}>0.05)$. The result of the platelet transfusion efficiency in terms of CCI value is presented in Table III. Effective rates of transfusion were 53 and $64 \%$ in groups I and II, respectively, and there was no statistical significance $\left(\chi^{2}=1.952, \mathrm{P}=0.162\right)$.

Therapeutic effect was evaluated according to clinical symptoms. There were 18 cases with bleeding tendency or symptoms in their oral cavity, nasal cavity, skin or vagina mucosa among 72 cases of group I, and 12 cases (67\%) had obvious improved symptoms after transfusion. While there were 20 cases who had bleeding tendency or symptoms among 83 cases of group II, and 12 cases $(60 \%)$ had obviously improved symptoms after transfusion. There was no statistical significance between the two groups $\left(\chi^{2}=0.041, \mathrm{P}=0.84\right)$.

There were 3 patients showing urticaria or fever reaction in group I (4.17\%) and 7 patients displaying similar reaction in group II $(8.43 \%)$ (Fisher's exact rates test, $\mathrm{P}=0.35)$.

\section{Discussion}

Platelet reduction can be induced by myelosuppression in treatments such as chemotherapy or radiotherapy. The problem of bleeding tendency or symptoms is particularly prevalent in blood disease patients caused by the disease itself. Platelet transfusions have been proven to prevent major haemorrhaging and improve survival in thrombocytopenic patients, and have now become an important method to guarantee continuous therapy in tumor clinical treatment (5). There are immune and non-immune factors that mainly influence transfusion efficacy (6). The former is due to the isoimmunization mainly caused by HLA antibodies and less frequently caused by antibodies for human platelet antigen (HPA) or a combination of HLA and HPA antibodies (7-11). Non-immune factors mainly involve the quantity and quality of infused platelets as well as some clinical factors such as fever, bleeding, sepsis, hepatosplenomegaly, disseminated intravascular coagulation and some medicine influence (12).

On the other hand, apheresis PCs are characterized by a superior haemostatic capacity over manual PCs both in vitro as well as in vivo (13-15). There are some reasonable advantages to single donor platelets including reduced disease transmission, reduced alloimmunization, superior function, and storage characteristics (16). Moreover, an equivalent dose of manual PCs requires at least 4-6 donors every time with increased alloimmunization, which is likely to cause platelet transfusion refractoriness (PTR). PTR is typically defined as failure to achieve an appropriate increment after receiving two consecutive transfusions with fresh ABO-compatible platelets. In the present study, manual PCs were found to somewhat exceed anticipation in terms of the platelet content and assessment of clinical efficiency. Furthermore, the rate of PTR was 36-47\% in our experimental research, versus earlier studies, which were 30-70\% (6). Patients in group II were superior to those in group I in terms of CCI values, but showed no statistical difference. The patients all showed good haemostatic capacity while group II was more likely to suffer from allergic reactions. Earlier findings, however, showed no difference between the two types of PCs according to clinical efficacy (17).

The following factors which have the ability to influence clinical efficacy should be considered. The quality of PCs is a major factor as lower quality PCs may directly result in PTR (5). Secondly, the clinical condition of each patient is different and complex, some patients are in the severe myelosuppression period during chemotherapy, which usually leads to a very low response after transfusion, while some patients are in the recovery phase of bone marrow therapy, which gives an active response to the transfusion with an obviously increased platelet count. Furthermore, the function and survival time of the infused platelet, and platelet-consuming factors such as bleeding tendency should also be considered. Therefore, we should always bear in mind that clinical efficacy is complex and variable because it is the result of the combined action and interaction of many factors. The results of the present study showed that manual PCs are more efficiently utilized in clinical setting in our area and hospital. However, manual PCs may generate more immunoreactions and allergies than APCs, but such methods as irradiation, leukocyte removal by filtration or HLA/HPA matching may be used to avoid the above side effects $(18,19)$. Furthermore, the proper usage of manually collected PCs could make full use of limited and precious blood resources.

\section{References}

1. Fletcher $\mathrm{CH}$, DomBourian MG and Millward PA: Platelet transfusion for patients with cancer. Cancer Control 22: 47-51, 2015.

2. Kumar A, Mhaskar R, Grossman BJ, Kaufman RM, Tobian AA, Kleinman S, Gernsheimer T, Tinmouth AT and Djulbegovic B; AABB Platelet Transfusion Guidelines Panel: Platelet transfusion: a systematic review of the clinical evidence. Transfusion 55: 1116-1127, 2015.

3. Schrezenmeier H and Seifried E: Buffy-coat-derived pooledplatelet concentrates and apheresis platelet concentrates: which product type should be preferred? Vox Sang 99: 1-15, 2010.

4. van der Meer PF: Platelet concentrates, from whole blood or collected by apheresis? Transfus Apher Sci 48: 129-131, 2013.

5. Holbro A, Infanti L, Sigle J and Buser A: Platelet transfusion: Basic aspects. Swiss Med Wkly 143: w13885, 2013.

6. Novotny VM: Prevention and management of platelet transfusion refractoriness. Vox Sang 76: 1-13, 1999.

7. Rebulla P: Refractoriness to platelet transfusion. Curr Opin Hematol 9: 516-520, 2002.

8. Pavenski K, Freedman J and Semple JW: HLA alloimmunization against platelet transfusions: Pathophysiology, significance, prevention and management. Tissue Antigens 79: 237-245, 2012.

9. Xia WJ, Ye X, Deng J, Chen YK, Xu XZ, Ding HQ, Luo GP and Fu YS: Study of the platelet GP specific antibodies and HLA antibodies expression in platelet transfusion refractoriness patients. Zhonghua Xue Ye Xue Za Zhi 31: 594-598, 2010 (In Chinese).

10. Eisenberg S: Refractory response to platelet transfusion therapy. J Infus Nurs 33: 89-97, 2010.

11. Hod E and Schwartz J: Platelet transfusion refractoriness. Br J Haematol 142: 348-360, 2008.

12. Shastry S and Chaudhary R: Clinical factors influencing corrected count increment. Transfus Apheresis Sci 47: 327-330, 2012.

13. Böck M, Rahrig S, Kunz D, Lutze G and Heim MU: Platelet concentrates derived from buffy coat and apheresis: Biochemical and functional differences. Transfus Med 12: 317-324, 2002.

14. Zebrowska A, Lipska A, Rogowska A, Bujno M, Nedzi M and Radziwon P: Quality of buffy-coat-derived platelet concentrates prepared using automated system terumo automated centrifuge and separator integration (TACSI). Pol Merkur Lekarski 30: 191-194, 2011 (In Polish). 
15. Yang M, Luo H, Shu B, An BQ, Xia SQ and Wang ML: Analysis on effectiveness of platelet transfusion in 1786 patients. Zhongguo Shi Yan Xue Ye Xue Za Zhi 21: 1038-1041, 2013 (In Chinese).

16. Kirkley SA and Blumberg N: Use of single donor platelets. Blood Rev 8: 142-147, 1994.

17. Cid J, Ramiro L, Escoda L and Llorente A: Efficacy of transfusion of platelet concentrates obtained by manual pooling or by semiautomated pooling of buffy-coats: a retrospective analysis of count increment, corrected count increment and transfusion interval. Vox Sang 96: 29-33, 2009.
18. Feng ML, Liu DZ, Shen W, Wang JL, Guo ZH, Zhang X, Du KM, Qian KC and Zhao TM: Establishment of an HPA-1- to -16-typed platelet donor registry in China. Transfus Med 16: 369-374, 2006.

19. Yuan Q, Chen X, Cheng L, Zhou CH, Fu XM, Li YP, Wang NH and Wang L: Prevention of platelet transfusion refractoriness and HLA alloimmunization by leukocyte filtered platelet transfusion: A meta analysis. Zhongguo Yi Xue Ke Xue Yuan Xue Bao 33: 412-420, 2011 (In Chinese). 